\title{
L'écriture de Mohammed Dib entre photographie et intertexte
}

\author{
Abdelaziz AMRAOUI \\ Université Cadi Ayyad Marrackech (Maroc) \\ a.amraoui@uca.ac.ma
}

Recibido: 10/02/2016

Aceptado: 08/04/2016

\section{Résumé}

En 1946, muni de son Rolleiflex, Dib, tout jeune, photographie sa ville natale Tlemcen. En 1994, il publie son livre Tlemcen ou les lieux d'écriture, un texte empreint de nostalgie et d'amour pour sa ville et pour son pays. Ses photos y côtoient ses textes anciens dans une biblio personnelle. Le lecteur se trouve entre un présent et un passé appelé sémiologiquement de deux procédés différents, mais complémentaires ; l'image et le texte. Dans cet article, nous nous proposons d'étudier comment la photographie s'est installée dans l'œuvre de Dib et comment elle se trouve au centre de son intérêt littéraire et nostalgique à la fois. Puis, nous verrons comment l'intertexte, devenu autotexte, est un moyen de vivre la nostalgie.

Mots clés : photographie, autotexte, intertexte, nostalgie, Tlemcen.

\section{Tlemcen ou les lieux de l'écriture de Mohammed Dib entre fotografía et intertexto}

\begin{abstract}
Resumen
En 1946, provisto de su Rolleiflex, Dib, muy joven, fotografía su ciudad natal Tlemcen. En 1994, publica su libro "Tlemcen o Lugares para Escribir", un texto impregnado de nostalgia y amor a su ciudad y a su país. Sus fotografías coexisten con sus textos antiguos en un libro personal. El lector se sitúa entre un presente y un pasado semiológicamente hechos de dos procesos diferentes pero complementarios: la imagen y el texto. En este artículo, nos proponemos estudiar cómo la fotografía se ha instalado en la obra de Dib y cómo se encuentra en el centro de su interés literario y nostálgico a la vez. Luego, veremos cómo el intertexto, convertido en autotexto, es una manera de vivir la nostalgia.
\end{abstract}

Palabras clave: fotografía, autotexto, intertexto, nostalgia, Tlemcen.

\section{Mohammed Dib's Tlemcen ou les lieux de l'écriture between photography and intertext}

\begin{abstract}
In 1946, equipped with his Rolleiflex, Dib, at a young age, took photographs of Tlemcen, his hometown. In 1994, he published his book Tlemcen or Places for Writing, a text marked by nostalgia and love for his city and country. His photos are to be found alongside with his older texts in a personal book. The reader is located between a present and a past semiologically made up by two distinct, but complementary strains: image and text. This article studies how photography is inscribed in the work of Dib, and how it
\end{abstract}


is located at the very heart of both his literary and nostalgic concerns. In his work the intertext, once it has become an autotext, is a way to live out nostalgia.

Keywords: photography, autotext, intertext, nostalgia, Tlemcen.

Sommaire : La mémoire et la photographie. La photographie dans l'œuvre de Dib. Photographie vs écriture. Le fragmentaire. Nostalgie entre écriture et photographie. Intertexte et souvenir. Conclusion.

Referencia normalizada

Amraoui, A. (2016). "L'écriture de Mohammed Dib entre photographie et intertexte ». Thélème. Revista Complutense de Estudios Franceses, Vol.31, Núm. 1: 9-23. http://dx.doi.org/10.5209/rev_THEL.2016.v31.n1.51671

En 1994, au sommet de sa notoriété, Dib publie, avec le concours d'un photographe professionnel, Philippe Bordas, Tlemcen ou les lieux de l'écriture, au diapason de ses parutions antérieures. Ce dernier visitera pour l'occasion les lieux de l'enfance de l'écrivain pour rephotographier les mêmes sites et lieux que Dib a fixés en 1946 avec un Rolleiflex qu'un ami lui a prêté. Les photographies vont remettre en mouvement une écriture qui s'est tellement diversifiée avec le temps pour retourner aux origines, aux «Lieux de l'écriture» (Dib, 1994 : 43) où l'écriture va aider dans la quête de soi et de toute l'Algérie de Dib. En se fondant sur un espace originel truffé d'images et de sensations, Dib montre et démontre à la fois que l'Algérie d'antan est quiétude, et celle d'aujourd'hui est inquiétude.

Le recours à la photographie rendra compte de cet éclatement mémoriel que Dib a cultivé durant tout son parcours d'homme de lettres. La valeur testimoniale, mais aussi mnémotechnique, font valoir leurs droits et contribuent à établir la reconnaissance d'un discours à partir duquel l'auteur inscrira son projet identificatoire, transportant son lecteur vers sa jeunesse, dans un élan de nostalgie au goût de fleurs de vent : «Merci, les anémones. Vous me rappelez tout à coup de vieux (comme ça, vieux, qui l'est sinon moi?) souvenirs » (Dib : $2003: 86$ ).

La photographie, dans Tlemcen ou les lieux de l'écriture, va être pensée en termes d'une opération de révélation d'un passé à double référence, le sien et celui de sa ville natale, et par extension de son pays, l'Algérie. Ainsi, pouvons-nous avancer que si dans la trilogie algérienne l'histoire, dans certaines de ses facettes, est basée sur la mémoire, Tlemcen ou les lieux de l'écriture, L'Arbre à dires et L.A trip, entre autres, par contre, sont fondés sur le mémoriel, cette trace qui va garder le souvenir intact et la mémoire vivante, et ce avec le concours de la photographie. 


\section{La mémoire et la photographie}

Les photos sont liées au passé et au souvenir comme une sorte de cristallisation de «possessions concrètes qui matérialisent la mémoire et, partant, la continuité » (Dagny, 2003 : 47) de la vie et de la nostalgie d'un passé meilleur, qui non contentes de fixer dans le présent l'écriture, la renvoient vers un passé presque idyllique. Les photographies vont remettre en mouvement une écriture qui s'est tellement diversifiée avec le temps pour retourner aux origines, à Tlemcen où l'écriture va aider dans la quête de soi et de toute l'Algérie. Elle va aider l'écrivain à cultiver sa mémoire. En fait, l'aventure littéraire entamée depuis 1952 sera à l'affût de tous les prétextes pour continuer à écrire. La photographie inspire à l'auteur, comme au spectateur, la rétrospection et l'envie d'écrire, surtout qu'elle paraît être «suspendue au souvenir projeté dans le futur de sa nostalgie» (Pivin in Dib, 1994: 7). Elle est comme l'autobiographie et l'autotexte, une fabrique de souvenirs qui tente de ne point perdre les repères du passé et «apparaît comme une extériorisation et comme une visualisation de la mémoire humaine » (Schuerewegen, 1999 : 16). Et réunies dans la même œuvre, elles reviennent à montrer ou raconter un double je, un je écrivain s'autocitant, et un autre photographe. C'est un moyen de montrer ce qui était ou ce qui fut et ceci ne se fait nullement sans conséquences. A ce propos Debray (1992: 44) dira que «L'image fait du bien parce qu'elle fait lien » entre un passé révolu et un présent en devenir.

\section{La photographie dans l'œuvre de Dib}

Nous commencerons par dire que l'action de photographier pour Dib est venue par accident. Il avoue dans L'Arbre à dires : «Rien dans mon enfance ni plus tard ne me destinait à devenir photographe » $(1998: 111)$ ce qui est très différent de peindre puis d'écrire : «A partir de douze ans, il est vrai, je me suis mis à peindre, et des années plus tard à écrire ; ce n'est pas la même chose. J'ai d'ailleurs abandonné la peinture peu à peu pour ne plus faire qu'écrire, jusqu'à aujourd'hui » (Dib, 1998 : 111). La photographie et la peinture, renvoient tous deux, du moins dans la conception la plus classique, à l'art figuratif. La peinture va préméditer un projet artistique futur, en commençant par effectuer une ébauche d'un déjà vu qui fournit au peintre la matière de la toile (pour ce qui est de la peinture réaliste ou figurative), alors que la photographie n'est que la réponse à une envie qui n'a duré que le temps de finir le film ou le cliché sans qu'il soit tenu compte à la concordance ou à l'affinité de ce moment avec un projet ou une idée préexistante : «La photographie, c'est l'action immédiate. Le dessin, c'est la médiation. [...] quand on dessine, on a son temps. Pas quand on photographie » (Assouline, 1994: 33). Dib restera essentiellement un écrivain. Et ce n'est qu'en 1994 que l'idée de faire paraitre un livre-album avait germé dans l'esprit de l'écrivain. Une distance temporelle sépare la prise photographique du commentaire consistant «à rapporter du langage à du langage » (Foucault, 1993 : 55). Alors que les clichés datent des années quarante du siècle 
dernier, les commentaires et les réflexions accompagnant leur publication datent respectivement de 1994 avec Tlemcen ou les lieux de l'écriture et 1998 avec L'Arbre à dires où Dib, dans «L'homme en proie aux images » donne expressément sa définition de la photographie qui rencontre de loin celle de Bresson :

La photographie capte l'instant et le fixe pour l'éternité. Là est le drame : elle assèche le temps, qui est expression de vie. Elle tarit tout ce qui, flux, s'écoule, passe, doit s'écouler, passer -- et dès lors ne va plus s'écouler, passer (Dib, 1998 : 107).

La photographie va à l'encontre du concept mécanique du monde. Elle immobilise un certain monde sous le signe d'un ça-a-été figé alors que celui-ci est une entité cinétique. L'instant photographique s'échappe fatalement. Et lorsque l'opérateur croit le saisir ou le capter, il s'est enfui déjà. Dans L'Arbre à dires Dib est en train d'introduire la littérature dans l'ère de la photographie, et c'est tout à fait le contraire de ce qu'il a fait dans Tlemcen ou les lieux de l'écriture où la photographie est ellemême littérature. Avec ce texte, Dib a changé de cap, et au lieu de commenter ses propres images comme c'est le cas dans son livre-album, il prend l'objet-photo pour thème. Il paraît que le projet de Tlemcen... s'organise autour du souvenir, d'un souvenir qui ravive la mémoire et extirpe le passé de sa passéité pour l'introduire dans le présent et le futur de l'auteur-narrateur-photographe, ce qui rejoint, et de loin, le noème de la photographie essentiellement «fabriqué[e] à l'instant dans toute sa vérité, étincelle qui, dès lors, fait que le monde n'est plus tout à fait le même » (Bacherich, 1990 : 139). Dans les photographies exposées, il y a d'abord un transbordement du passé vers le présent. La photographie, pour ainsi dire, va saisir un instant présent et fatidique dans son immédiateté, mais va l'inscrire illico presto dans sa densité du passé. Pourtant, ce long retard ou ajournement est manifestement cohérent avec l'esprit de l'art photographique qui fonctionne, principalement, avec le reg(t)ard et la distance. En effet, en retournant dans son passé par le biais des photographies qu'il a prises, l'auteur met l'index sur une particularité de l'arsenal photographique, qui se base essentiellement sur l'entrée en force du passé dans le présent avec toujours le décalage temporel qu'il exige. En ce sens, la photographie est inchoative. Elle fait passer le cela-est en un cela-a-été et rien ne peut arrêter cet état de chose. D'ailleurs, dans le même recueil d'essais L'Arbre à dires, Dib dit que l'appareil photographique grâce auquel il a eu ses prises lui a été prêté : «... un ami marchand de matériel photographique, m'avait prêté à l'époque un appareil, un Rolleiflex. A ce moment-là je n'avais même pas de quoi acheter de la pellicule» (1998:112). Cet appareil a particulièrement servi aux street photographers et a «été tardivement utilisé dans la rue, son système de visée particulier permettant d'une certaine manière de voir sans être vu » (Bauret, 1991 : 58) établissant deux distances distinctes, celle par rapport au sujet de la prise et celle entre les yeux du photographe et de son appareil. 


\section{Photographie vs écriture}

La photographie dans Tlemcen ou les lieux de l'écriture est un moyen pour plonger dans le passé, tous les passés de Dib. L'enfance, la littérature, la ville, les anecdotes, l'écriture, la famille, la rue... tout viendra hanter littérairement (donc positivement) l'esprit de l'auteur et donner le postulat de sincérité que le lecteur est prêt à accepter. L'auteur-narrateur-photographe part donc à la recherche de soi et des siens et retrouve ainsi la mémoire. Ce n'est pas un album à découvrir à tâtons ou à l'aveuglette. Les mêmes yeux qui ont visé, pourtant vieillis de 40 ans, et la même main qui a déclenché le tir, vont nous organiser une visite guidée dans le labyrinthe de ces passés par les commentaires et les textes accompagnant les images, dont la conjonction formera l'iconotexte que nous pouvons définir comme étant un rapport de co-présence entre le texte écrit et l'image, par la présence matérielle de l'un dans l'autre, que Liliane Louvel appelle (1988) «l'œil du texte» permet un flash-back retrouvant ainsi les racines de toute chose dans la vie de l'auteur: famille, écriture, ville, photo... créant une nette coupure dans la tradition littéraire maghrébine habituée à passer de la parole pour trouver une image et non pas l'inverse.

L'image devient donc le prétexte à écrire, et le commentaire «sera l'équivalent de la planche de contact, chaque [phrase] "captant" un fragment de réel, de réel écrit, par l'acte du cadrage... » (Gleize, 1983 : 254). D'ailleurs, le commentaire en tant que reformulation de l'image par le subterfuge d'un autre système sémiologique, est un autre révélateur qui dit la vérité, au sens photographique du terme. Hiérarchiquement, ceci donnera la prééminence à l'image, du fait que «le signe », qui, comme le divan psychanalytique, va chercher derrière la tête de l'auteur pour trouver les souvenirs enfouis dans la mémoire et « [révéler], à partir d'infimes incidents, comme un lapsus, une pulsion refoulée » (Ortel, 2001: 304) qu'il suffit seulement d'activer et tout apparaîtra : "Le signe a partie liée avec la mémoire pour sûr, mais sans être la mémoire, il est ce qui agit sur la mémoire sans se confondre avec elle, la mémoire étant ce qui est là, toujours présent » (Dib, 1998 : 196).

Comme étendu sur un divan psychanalytique, le patient va étaler son moi, et ouvrir sa boîte de Pandore à partir d'un point de repère situé loin dans le temps, et c'est exactement ce que la photo, toutes les photos de Tlemcen... exercent sur l'auteur, qui va se trouver dans l'obligation de plonger dans le passé. D'ailleurs, étaler veut dire exposer, déballer, montrer, dérouler, déployer, voire offrir au regard. L'étalement du corps sur le divan nous rappelle curieusement et à notre insu à la fois la platitude de la position allongée dont Régis Debray (1992: 119) parle : «La lettre redresse, l'image allonge (nos plus belles images, nous les voyons couchés, et se vautrer dans son fauteuil est un plaisir cinéphilique) ». Et quand la photo se fait, elle opère une saisie dans le temps en mouvement et l'arrête en conséquence. L'écrit est donc une parole allongée, couchée sur un support-papier qui assurera à la parole et à l'image leur mouvement en tant que principe de transcription et catégorie indispensable pour la signifiance. D'ailleurs les photographies ont un rapport intrinsèque avec le hic et le nunc du producteur, et deviennent déclencheurs de mémoire en tant que signe, 
exactement comme pour une image «Car l'image fabriquée est à la fois un produit, un moyen d'action et une signification » (Debray, 1992: 111). L'étalement ou la platitude va rendre visible le penser.

Le signe donnera une amplitude à la mémoire de telle façon que notre recueil se trouvera cantonné dans le fait de raconter comment les lieux de l'écriture ont façonné l'écrivain. Le lieu, en fait, tout lieu comme la maison ou même la mosquée, sera à l'origine d'un autre lieu dans du papier où l'auteur va coucher ces récits/écrits, et Tlemcen, le chef-lieu de tous les lieux y figurant va fonctionner comme le bain révélateur qui animera la mémoire de l'auteur. Et au-delà des relations classiques qu'entretient l'image avec le texte écrit, telles la complémentarité, la répétition ou la contradiction, une autre relation s'installe. Celle-ci s'articulera sur le vœu non prononcé de l'écrivain d'étouffer toute lecture autre que la sienne des images prises de Tlemcen en faisant emporter la primatie du mot sur l'image. Et même si l'image prend depuis longtemps de l'ampleur dans la «logosphère » rien ne peut remplacer les mots. D'ailleurs, si Dib a renoncé à la photographie pour une raison ou une autre, il reste devant elle comme un éternel amateur, voire comme un éternel amoureux. Du fait, vite il va en quelque sorte troquer l'appareil photographique pour la plume.

\section{Le fragmentaire}

La photographie, telle qu'elle est vue par Dib, célèbre le fragmentaire et le parcellaire. Elle ne peut que rendre une restitutio in integrum du moment. Il est vrai que le traitement de l'information ou des faits sur un tel support diffère beaucoup de l'écriture. L'écriture qui s'étalant, par petits fragments, mot après mot, phrase après phrase, donnera à la fin une totalité compacte. Par contre, la photographie, totale et immédiatement donnée au spectateur à première vue, est fragmentaire dans son essence. Elle isole une partie de la réalité et installe dans une vraisemblance en trace le visible. La copie ainsi obtenue rendra compte d'une réalité plus complexe et plus large que cela paraît. Dans le figement forcé du cadre photographique, le monde se livre par fragments, qui se mettent à le clamer en son entier. Elle livre au regardeur ou focalisateur une surface oculaire prête à déborder d'un cadre dont les mots viendront combler un manque originel : le hors-cadre, domaine d'un fragment perdu du temps et de l'espace du monde référentiel. En fait, pour toute image visuelle, des zones de lumière (le cadre lui-même) côtoient, et ce imaginairement, des zones absentes. Barthes (1980 : 18), à propos de la photographie dira : «Quoi qu'elle donne à voir et quelle que soit sa manière, une photo est toujours invisible : ce n'est pas ce qu'on voit ». Et c'est au récit que revient la lourde tâche de combler ce déficit perceptif en parlant des conditions générales dans lesquelles la prise de vue a été tirée. Ceci peut ressembler, et de loin, aux procédés autobiographiques qui, faute de ne pouvoir raconter tout, se laissent tenter par opérer des choix dans le cours de la vie du narrateur, et amputent en conséquence des épisodes. Il s'agit d'une atomisation aidant l'auteur ou le narrateur à mieux reconsidérer et reconstituer son passé à base de simples fragments situés ci et là dans le temps et dans l'espace. 


\section{Nostalgie entre écriture et photographie}

L'écriture s'abreuve à la source d'une espèce de nostalgie originelle, nostalgie douce, complainte lancinante qui berce et ravive les sensations primaires, et vite devient une envie frileuse pour récupérer le passé de l'oubli et de l'érosion mnésique. Le titre même de son album invite le lecteur-regardeur à l'exploration de son passé dans sa version non moins autobiographique, mais aussi photographique. Littéralement, nostalgie est «douleur du retour » ou comme le dit Dib : «effet de retour qui joue ici à la manière du piège dans sa figuration symbolique de circuit fermé, de cercle vicieux » (Dib, 1998: 62) dans laquelle l'auteur garde caché en lui le pressentiment d'une représentation heureuse (son enfance à Tlemcen).

Rien de mieux, de plus captant que de faire plonger la nostalgie dans les «Grands yeux noyés de nostalgie » (Dib, $1984: 47$ ) qui par le concours des circonstances, sont les premiers à souffrir de la séparation et de la rupture. Tout le toujours-vu deviendra, hélas, un in-vu dans le présent, un déjà-vu dans le passé. La coupure, ou la distanciation est un fait avéré dans le processus de la représentation car la référence est référence à quelque chose, par essence, détachée organiquement de celle-ci. Ainsi, la consultation des photographies viendra-t-elle « annuler les effets de la séparation » (Tisseron, $1987: 50$ ) par cette opération consistant à nommer et à constituer un objet introjecté en un objet montré et détaché, d'abord de soi, du fait qu'il n'est plus qu'un objet mémoriel, mais aussi un objet entré dans le domaine public. Mais, toujours est-il que le signe ou le référent va essentiellement renvoyer à l'objet qui en a donné vie par procuration. Plus de contact visuel, sauf, parfois, face à une photographie qui rendra présent ce que les yeux ont perdu d'où la naissance d'un sentiment douloureux : « Là où il y a douleur, c'est l'objet absent, perdu, qui est présent ; l'objet présent, actuel, qui est absent » (Pontalis, cité in Scarfone, 1993 : 17). Pour Dib, cet objet était, et ce depuis l'action de photographie, du domaine du privé et rien ne le prédestinait à entrer dans le domaine public comme c'est le cas de sa vie narrée et re-narrée dans ses récits. Et notre objet accepte les deux qualificatifs et parait être un objet ambigu de par ses effets. Il est «empreinte douloureuse » et du coup, « la douleur de séparation apparait comme secondaire à une douleur nue, absolue » (Freud, 1981 : 101) qui enverra Dib dans un «état zéro», un trou noir: «On ne part jamais de l'état zéro, on y aboutit. Présence de l'absence, tache aveugle, blancheur. L'état zéro » (Dib, 1998 : 25). Chez lui, la photo, essentiellement objet, est liée à un souvenir, une réminiscence jubilatoire qui non contente de fixer dans le présent l'écriture, la saisit vers un passé nostalgique, donnant à l'auteur l'envie d'aller très loin dans son passé, dans le temps mais aussi dans l'espace : «L'appel dès lors, accompagné de nostalgie, s'élèverait de là-bas, où il a noué des liens, contracté des attachements tout neufs, inédits » (Dib, 1998: 67).

En fait, l'auteur va entamer une régression dans son histoire et celle de sa ville en révélant un contenu de vérité contre l'absurdité de la réalité algérienne après «Les Soleils des indépendances » comme dirait Ahmadou Kourouma. En effet, le monde de l'enfance vient habiter le monde de l'homme adulte. Cela ne peut pas seulement 
être un simple retour nostalgique, mais une sorte de pèlerinage vers un espace-temps élevé à un rang, somme toute, meilleur que ce que vit l'Algérie des années 90 caractérisées par la recrudescence du terrorisme et la montée de l'obscurantisme : «le monde, c'est regrettable à dire, change de visage sans prévenir. Ce qui est devant vous se met subitement derrière, ce qui est ouvert se ferme ; blanc se fait noir, près se fait loin» (Dib, 1994 : 112) contrairement à la période de la prise des photos contenues dans Tlemcen... où «Toutes [les] images d'avant le déluge vous chantent pourtant plus qu'aucunes autres une petite musique douce-amère. C'est à fondre de nostalgie» (Dib, 2001: 166). Avec la nostalgie, le lecteur de Dib ne peut que constater que Tlemcen ne peut être envisagée que comme un passé doré mais perdu.

Concrètement, Dib a recours à des images simples et tendres telles que nous les voyons dans Tlemcen... (la famille, la rue, les enfants...) pour illustrer les oppositions aux oppressions et à l'hypocrisie, en élevant la ville en un vrai lieu de délice et de délectation. Elle est l'alpha et l'oméga de tous les topophiles, de tous les espaces heureux.

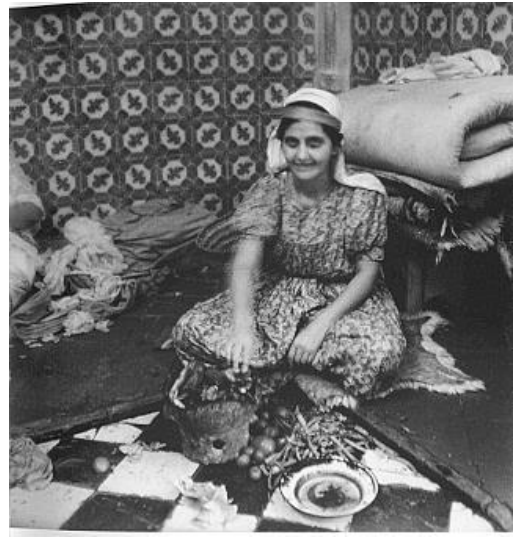

(Photo sans titre)

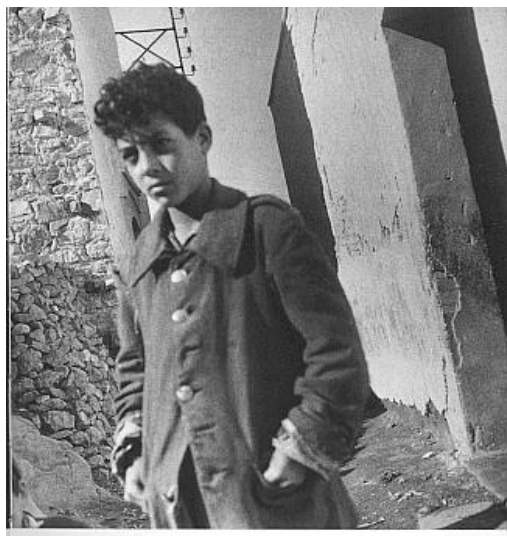

(Photo sans titre)

C'est une ouverture, une brèche, pour faire un travail de deuil. Un deuil pour toujours vécu, et en s'accomplissant, l'individu se constitue. Et ce sont ces photos qui vont permettre à l'auteur, dans son exil volontaire, de visiter ses racines via une brèche rappelant une tombe exhumée : «... l'ouverture n'aura pas encore lieu sans le travail de deuil long, secret, que le sujet aura à effectuer et dont parfois on ne se rend pas compte. Seulement alors tous les espoirs seront permis » (Dib, $1998: 66$ ).

C'est un deuil d'un certain être, et non de toute une identité, avec tout ce que ce vocable comprend comme éléments intrinsèques tels que la culture et l'appartenance à une terre. Le passé depuis longtemps enfoui dans un cercueil comme s'il s'agissait d'un mort s'ouvre et devient béant pour offrir au lecteur tous ses secrets. Dib en deuil est martyrisé et se voit dans l'obligation de s'exprimer d'une façon ou d'une autre. Parfois ouvertement et en parlant par et dans ses entretiens ; parfois poétiquement dans 
ses récits et ses vers; cette fois avec ses photographies, qui vont, comble de paradoxe, faire parler «le néant qui s'envisage, se fait une tête pour [...] faire face [au regardeur] » (Dib 1998 : 109) (L'italique est de l'auteur).

En ce sens Tlemcen... offre la possibilité de traiter du sujet du retour aux sources, hélas ! perdues depuis que l'auteur a choisi la voix/e de l'exil. D'ailleurs, dans une tautologie forcée, le vocable «Tlemcen » signifie pays de sources, «son nom même l'annonce d'entrée de jeu. Issu du berbère tilmas, au pluriel tilmisân, il veut dire sources » (Dib, 1994 : 109). Et en revenant aux sources (à Tlemcen la ville) Dib joue soi-même la pérennité en évoquant la vie. En fait, quoi qu'il en soit, le passé est vie, et le futur, ce temps virtuel, est mort puisqu'il contient irrémédiablement la mort. Tel est le fatum et tel aussi ce que Le Dictionnaire de symboles (1982 : 904) stipule : "la source symbolise l'origine de la vie, et d'une façon plus générale, toute origine, celle du génie, de la puissance, de la grâce, celle de tout bonheur ». Bon gré mal gré, la photographie de Tlemcen, comme d'ailleurs toutes celles qui lui ressemblent dans leur essence, n'est ni plongée dans le passé, ni prolongement dans un futur absolu, elle est les deux à la fois. Elle s'installe sur l'axe du temps et va dans les deux directions. Cette œuvre symbolise la «REPRESENTATION» (Tlemcen... : ) avec des lettres capitales. Plus que la mise en exergue, cette graphie illustre, aussi, la mise en abyme du mot lui-même dans un contexte iconotextuel. Cette graphie doit être prise comme le correspondant typographique du caractère suprasegmental d'une intonation qui accentue un fragment phrastique. D'ailleurs, le livre-album tourne autour de celle-ci, en faisant de la photographie, de l'autographie, de l'intertexte des instruments intermédiaires qui tiennent lieu de leur objet. Les renvois intertextuels, ainsi dit, s'érigent en monuments. Ils établissent un rapport entre passé et présent dans une continuité littéraire et personnelle. Tlemcen... devient ainsi l'alpha et l'oméga d'une trajectoire qui a mené l'auteur sur les routes de l'errance, toutes les errances qu'elles soient géographiques ou littéraires. Il est par où tout a commencé et vers quoi tout mène, et dont les textes renvoient éternellement à d'autres textes par le subterfuge de renvois et de la citation, définie par Antoine Compagnon (1979: 34) comme étant «lecture et écriture ».

\section{Intertexte et souvenir}

À l'intérieur du système intertextuel, nous rencontrons chez Dib l'autotextualité. Elle fait jaillir le souvenir des textes anciens dans des textes postérieurs présentés comme un vécu déjà plusieurs fois transformé par ses retranscriptions successives Les souvenirs des textes, le texte de mémoire (autobiographie) et la mémoire des textes (intertexte et intratexte formant l'autographie) se recoupent jusqu'à ce que l'autobiographique devienne autographique. Cette intertextualité aidera le lecteur à épuiser les possibilités sémantiques de la phrase et du texte par extension. Cela rejoint, et de loin, la pensée borgessienne qui voit le travail d'un écrivain comme une saisie d'une représentation déjà représentée. Du fait, la narration ou la diégèse virerait plutôt vers le commentaire. Désormais avec l'intertexte c'est la mémoire textuelle qui 
fonctionnera pour arriver à cette réalité épistémologique : le texte dibien n'est guère un produit arrêté, il est un prétexte pour le nouveau qui boira de l'ancien comme d'une source-mère abolissant la lisière entre eux «particip[ant] ainsi à l'appropriation symbolique des fragments d'expérience antérieure » (Tisseron, 1999: 86). Le texte va survivre en le replantant dans un texte présent. Le nouveau récit est un recommencement des autres, une création pour toujours répétée : «Vous n'avez ni tout dit, comme vous avez cru l'avoir fait, ni bien dit ce que vous aviez à dire. [...] Tenter à nouveau l'aventure » (Dib, $1994: 72$ ).

De ce qui vient d'être avancé, il devient possible de poser l'intertexte et l'autotexte comme équivalents scripturaires des atlal ${ }^{l}$ que l'on visite afin de ne point oublier le passé, partie intégrante du présent. Les atlal ou vestiges sont aussi symbole du monde dans lequel vivent et les personnages et les lecteurs et qui rendent compte de l'anéantissement de toutes les normes et de toutes les règles, fût-ce littéraire, et plus particulièrement romanesque.

Une certaine lecture voit le jour. Il s'agit d'une lecture guidante que l'intertextualité va prendre en charge puisqu'elle est « un phénomène qui oriente la lecture du texte, qui en gouverne éventuellement l'interprétation et qui est au contraire de la lecture linéaire » (Riffaterre, 1981:5-6). Il s'agit d'une lecture qui va au-delà de ce qui est lu dans l'immédiat, pour aller vers un biblon personnel plus large en citant ses propres œuvres: Le Sommeil d'Eve qui fait appel à des référents intertextuels explicites comme le Coran et Ibn Arabi (Averroès) d'un côté ; et évoque d'imminents chercheurs en langage et mathématiques comme c'est le cas avec Carnap, Wittgenstein et Freege; du Désert sans détour, où l'écriture et le thème renvoient à En attendant Godot de Beckett et à Don Quichotte de Cervantès, dans une intertextualité proche, plutôt, de l'allusion ou du clin d'œil avec quoi une opération d'identification peut avoir lieu avec un texte antérieur, laissant paraître comme dirait Riffaterre (1981: 5) une «trace [...] indiqu[ant] la présence latente, implicite, d'un corps étranger, qui est l'intertexte». Dib n'a nullement peur d'étaler sa culture universelle dans ses écrits. C'est ainsi qu'il se balade d'une culture littéraire à une culture théologique, passant par divers sentiers qui rappelle les cultures scientifique, technique, philosophique, photographique, sans oublier au passage de faire une halte pour se ressourcer des théories psychologiques et psychanalytiques... pour écrire un récit décentralisé avec une grande liberté loin de l'univers du récit avec toutes ses exigences. L'écriture devient une pratique loin des codes du discours dans laquelle elle a été depuis longtemps cantonnée. Ainsi dit, l'intertexte n'est pas seulement affaire d'un biblon scripturaire, mais, il la dépasse pour toucher la sphère iconique et visuelle. Et si le $\mathrm{XX}^{\text {ème }}$ est celui de l'image, il est aussi celui du regard, et que Dib exploitait dans ses écrits. Il est devenu un être doué de curiosité et de sens, dans un

\footnotetext{
${ }^{1}$ Atlal est un nom arabe, pluriel de talal qui veut dire ruine ou monuments en état de ruine.
} 
monde qui se cherche : «La traversée de culture à culture n'est pas d'une difficulté surhumaine, il suffit de vouloir l'entreprendre, et l'on découvre que c'est une aventure passionnante. Alors sera passé le temps où la préférence joue uniquement en faveur des œuvres-documents à toile de fond ethnographique, voire folklorique » (Dib, 1998 : 17). Dans le cas de Tlemcen..., le biblon est quasiment dibien, répétant in texto des fragments de son passé littéraire afin de les apprivoiser et de ne plus s'en détacher. Il s'ensuit qu'au sein de ses écrits, quels qu'ils soient, nous sommes devant une question qui touche et de près le sujet de la réflexivité et de du jeu de miroir, qui sont ceux de la fictionnalité et de la référentialité des textes dibiens. L'autotexte donne à voir le patrimoine littéraire de l'auteur sous une couverture de monument apte à être visité, vu sa valeur littéraire et testimonial.

L'histoire de la vie devient une histoire des écrits donnant «à lire et à voir des morceaux de genèse, réactualisant les récits » (Bererhi, 1985 : 135) de la naissance d'un écrivain. Du fait, deux temps vont se mêler jusqu'à la fusion : le temps de la mémoire ou le temps mnésique et le temps de l'imaginaire. Ceci va ranger Dib au titre des lecteurs de ses propres créations : "L'écriture [...] est une lecture, et toute lecture est lecture de soi, le sujet du texte [...] doit être le seul sujet-lecteur-de-soi » (Bal, 1997: 59). Cette forme intrinsèque d'intertextualité répond à l'idée de la fixation dans le temps des productions antérieures dibiennes puisque basées surtout sur l'autocitation, forme de miroir textuel recontextualisant les textes anciens considérés comme une perception de soi par soi laissant dégager un savoir intérieur latent. L'autocitation, dans ce sens va être considérée comme une re-découverte de soi et une stratégie qui lie le passé au présent dans une espèce $\mathrm{d}^{\prime}$ ' absorption et [de] transformation d'un autre texte »(Kristeva, 1969 : 146). Pour sa part, Dib dira que

Ceux qui ont eu la curiosité de me lire pourront en témoigner. De l'un à l'autre de mes livres, des passerelles sont jetées, non d'une manière calculée mais comme la conséquence naturelle d'une manière de procéder, traverses qui relient chaque livre à un autre, nullement dans une succession logique, mais organique. Car ce n'est pas une suite romanesque, ou poétique, que je me suis efforcé de mettre sur pied, j'ai été tenté au contraire par l'aventure que constitue une exploration tous azimuts... Aussi des personnages, des lieux réapparaissent, des situations se recréent. Peut-on vraiment parler d'avancées par récurrences ? Je me le demande (1998: 208).

Ces propos inscrivent le projet littéraire de l'auteur dans une dynamique intertextuelle. Non seulement les textes se renvoient, comme dans un jeu de miroir, les thèmes, les personnages (Lylli par exemple), mais ce sont des phrases complètes reprises in texto que nous rencontrons ici ou là dans ses textes comme pour Tlemcen ou les lieux de l'écriture. Ce projet est à l'image d'une œuvre musicale qui va jouer sur les variations dans lesquelles les portées musicales se suivent, se répètent sans jamais nous lasser de les apprécier.

Le narrateur semble être en perpétuelle recherche de son passé, de tous ses passés, personnels et littéraires ou créatifs, qu'il devient comme l'a expliqué Mieke Bal (1997 : 10) dans son travail sur Proust «focalisateur» sur tous les états de son soi. L'histoire de son Histoire est donc toujours à réécrire et à revisiter. Toutes les 
occasions sont bonnes pour plonger dans le passé afin de s'autoreprésenter. D'ailleurs, le souvenir ou la remémoration de quelques lignes de textes anciens équivaudrait à plusieurs égards à revoir une photographie prise une quarantaine d'années auparavant, puisque, dans le premier comme dans le deuxième cas, l'auteur est devant le déjà $(+$ participe passé) vu, lu ou écrit. Pour le reste, «[la citation] a une fonction [...] de mémorabilité » (Compagnon, 1979: 91). L'auteur procède à l'appel des informations d'un monde ancien, son passé littéraire et sa mémoire qui à l'occasion rend l'oubli désuet. Le monde antérieur est omniprésent. Il devient donc aisé de constater quel rôle joue le rappel à l'ordre de l'effet autobiographique via l'effet autographique et vice-versa. Ainsi, un ouvrage est toujours l'occasion d'inscription d'autres textes tirés d'écrits antérieurs dans une quête de restauration du déjà lu pour une potentielle réactualisation. Et en se répétant, une dimension métatemporelle s'instaure par le fait de la sollicitation du passé dans le présent. La fusion des deux temps dans une espèce de boîte de Pandore où, d'ailleurs, l'écriture et la lecture sont réunies, va créer ce que nous pouvons appeler le procédé d'écriture-lecture-écriture. Ce procédé est basé sur une rumination du fait du retour de l'auteur sur ce qu'il a écrit pour l'aider dans son écriture actuelle. D'autre part, cette répétition maintient le régime de la lecture dans l'écriture. Tlemcen.ou les lieux de l'écriture devient le noyau d'un réseau intratextuel convoquant tour à tour L'Incendie ${ }^{2}$, Au café ${ }^{3}$, Qui se souvient de la mer ${ }^{4}$ et Le Désert sans détour ${ }^{5}$. Dib semble avoir ouvert une brèche où il a pu s'immiscer dans son passé, tout son passé comme s'il ouvrait ses archives pour consultation. Au total nous recensons 5 renvois à 4 œuvres antérieures très espacées dans le temps, dans une espèce d'écriture de son écriture. Ces reprises respectent le texte primaire d'où chacune des autocitations sont tirées, et intègrent un espace de sens autre que celui qui leur a été d'abord assigné. La fidélité du premier texte est de rigueur. Elles se comportent au sein du texte accueillant comme des textes photographiés et collés dans un autre texte. Ce procédé ressemble à la compilation musicale où l'on rencontre des artistes différents réunis dans un même album thématique. Tlemcen..., et de loin, connaît le même phénomène, mais il rassemble dans une compilation thématique que nous appellerons «les nostalgiques» des fragments autotextuels anciens rappelant une mosaïque de sources sui-génériques. D’un contexte diégétique dans le cadre d'une fiction, les reprises trouvent un cadre différent où elles ont le rôle de renvoyer aux «lieux de l'écriture » et au passé. Des fragments littéraires du même auteur deviennent des fragments réécrits, alors qu'une grande distance temporelle les sépare. Ces intertextes jouent un rôle important quant à leur régime fictionnel dans un contexte référentiellement autobiographique. Tlemcen... inverse les rôles des créations

${ }^{2}$ L'Incendie, Paris, Le Seuil, 1962, pp. 58-60.

${ }^{3}$ Au café, 1956, p. 86.

${ }^{4}$ Qui se souvient de la mer?, Paris, Le Seuil, 1962, 1990, p. 90 et p. 125.

${ }^{5}$ Le Désert sans détour, Paris, Sindbad, 1992, p. 108. 
littéraires en faisant précéder la représentation du réel au fait de créer un monde fictif qui feint la réalité ou le vécu. Les autocitations renvoient expressément au temps de l'écriture et au temps de la réception de l'œuvre ou des œuvres en question et s'approchent de la notion qu'a élaboré Barthes quand il dit de son Roland Barthes par Roland Barthes (1995: 327) que «C'est du romanesque intellectuel [...] Je me suis mis en scène comme un personnage de roman, mais qui n'aurait pas de nom propre, en quelque sorte, et à qui il n'arriverait pas d'aventure proprement romanesque ».

Et si comme Barthes ou Duras, Dib n'apparaît pas dans ses photos contenues dans son album, il est arrivé quand même à trouver le détour par quoi il va se manifester. Et c'est, d'abord, dans une affirmation de soi à travers une mise en scène textuelle avec l'emploi de «je» autobiographique et l'intratexte, que cela s'est réalisé. Pourtant, il faudrait voir dans Tlemcen ou les lieux de l'écriture..., non seulement un retour au soi, mais aussi un retour à sa ville latine, à son pays.

Ce sont des éléments attestant un fait avéré, comme c'est le cas de ses photographies de Tlemcen... Ontologiquement, les deux, à savoir l'œuvre littéraire et la photographie, ont existé et continuent d'exister et de faire irruption dans le présent. L'intertexte est aussi un passé présent comme la photographie. C'est un retour en arrière à la rencontre d'une création langagière.

Sur un autre plan, Tlemcen... est construit sur le mode d'une analepse par rapport à l'œuvre de Dib surtout que celle-ci est un concentré d'œuvres antérieures d'une part, et un document historique dont la véridicité est à chercher dans le témoignage des photos, des années d'enfance et d'adolescence de Dib. Ainsi la photographie chez lui serait-elle cette chose «irrécusable, indemne de toute contamination humaine » (Brassaï, 1997 : 152), elle est

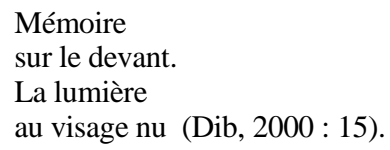

Et comme la photographie dibienne est centrée sur le souvenir et le retour nostalgique aux sources, Tlemcen ..., du moins sa partie textuelle avec tout ce qu'elle comporte d'autocitations, «appartient à l'origine, elle est souvenance de l'origine » (Compagnon, $1979: 34)$.

\section{Conclusion}

La photo devient dans l'œuvre de Dib un moyen de communication en force. Elle traite un sujet de politique et de société d'actualité, avec des images d'antan. Son but est de communiquer un certain «avoir-été » en le ramenant à l'expérience vécue de l'auteurnarrateur-photographe «pour se retrouver dans l'ouverture inconfortable d'un univers désormais flottant » (Didi-Huberman, 1990 : 172). Elle devient, comme par miracle, un élément essentiel pour un projet didactique, qui prend le passé comme repère et lieu de comparaison en s'adressant directement à l'esprit à travers des images matérielles 
données à voir, qui vont en définitive réussir à libérer les consciences de la peur et de l'angoisse du contemporain. L'Algérie, dans ses aspects historiques (passé, présent et futur), devient, d'une part, paysage ouvert à la contemplation et à la lecture du monde, et assure, d'autre part, la diffusion de sa voix et de son image par Dib, parce que tous les deux ne font désormais qu'une seule entité ontologique. L'un reflète l'autre : «D'abord, entre mon horizon et moi, le partage n'est pas fait. Lui et moi ne savons pas encore qui est l'autre. Puis nous le savons. Et dans le recul pris, le paysage, identique dans sa pérennité, s'érige en témoin des origines » (Dib, 1994 :43).

En nous guidant dans Tlemcen par le subterfuge des photographies et de l'autotexte, que nous considérons comme un intertexte endogène dont la grande particularité est la reprise, c'est en lui-même que Dib nous promène. Avec l'iconotexte, Dib est avantgardiste, il prend les photos de Tlemcen la ville comme archives visuelles d'où va se déployer un discours sur le moi, sur l'écriture, sur la ville... dans une espèce de registre rappelant le documentaire social où la devise est « Photographier en citoyen, penser sur le réel, infléchir le cours-du-Monde fondent le credo des photographes [lui] appartenant » (Gaessler, $1995:$ 54).

La photographie devient donc non seulement un objet à contempler, pour apprécier son apport artistique ou amateur, mais un tremplin de construction sémiotique amplifiée par un apport de sens indéniable. Tlemcen ou les lieux de l'écriture va permettre à son auteur de recontextualiser son pays en lui donnant un autre visage que la communauté internationale commence à estomper au profit d'images récentes. Le recueil utilise des photographies anciennes pour construire une image positive de l'Algérie dans une période de crise sociale et identitaire. Du fait, la mission de la photographie, rejoindra, et de loin, la fonction de l'art, que George Sand décrivait dans «L'auteur au lecteur » de La Mare au diable, quand elle a stipulé «que la mission de l'art est une mission de sentiment et d'amour [...] , et que l'artiste a une tâche plus large et plus poétique [qui] devrait être de faire aimer les objets de sa sollicitude, et au besoin, je ne lui ferais pas un reproche de les embellir un peu » (Sand, 1989: 31). A côté de la photographie, l'intertexte dans toutes ses configurations, a rendu compte de la continuité et de l'évolution de l'écriture de Dib, toujours à l'affût de nouvelles expériences scripturales.

\section{REFERENCES BIBLIOGRAPHIQUES}

Assouline, P., (1994) « Henri Cartier Bresson », Entretien in Lire. $\mathrm{N}^{\circ}$ 226-227, pp. 30-37.

Bacherich, M., (1990) Je regarde Manet. Paris, Adam Brio.

Bal, M., (1997) Images littéraires, ou comment lire visuellement Proust. Montréal, XYZ. Barthes, R., (1975) Roland Barthes par Roland Barthes. Paris, Seuil.

Barthes, R., (1980) La Chambre claire, Notes sur la photographie. Paris, Gallimard, Cahiers du cinéma.

Brassaï, Gy. Halàsz (dit), (1997) Marcel Proust sous l'emprise de la photographie. Paris, Gallimard.

Bererhi, A., (1985) «Aux voix(es) du désert: Le Sens », in Itinéraires et contacts de cultures. Vol. $21-22,1^{\circ}$ et $2^{\circ}$ semestres, pp. 133-138. 
Bauret, G., (1991) «Formats » in Photographies Magazines. $\mathrm{N}^{\circ}$ 35, pp. 58-65.

Compagnon, A., (1979) La Seconde main, ou le travail de la citation. Paris, Seuil.

Dagny, I., (2003) «L'arbre des Gratiolet ou les déboires du marquis de Carabas (étude sur la famille Gratiolet dans La Vie mode d'emploi de Georges Perec) », in Littérature, " Masques et intertextes ». $\mathrm{N}^{\circ} 131$, pp. (37-58).

Didi-Huberman, G., (1990) Devant l'image. Paris, Minuit, Coll., critique.

Debray, R., (1992) Vie et mort de l'image. Paris, Gallimard.

Foucault, M., (1993) Les Mots et les choses, Une archéologie des sciences humaines. Paris, Gallimard, Coll. Tel.

Freud, S., (1981) Inhibition, symptôme et angoisse. Paris, P.U.F.

Gaessler, D., (1995) «Pièces à conviction, A propos du documentaire social», in Photographies Magazine. №65, pp. 54-55.

Gleize, J-M., (1983) Poésie et figuration. Paris, Seuil, Coll. Pierres vives.

Louvel, L., (1998) L'Eil du texte. Texte et image dans la littérature de langue anglaise. Toulouse, P.U.M., Coll., Interlangues, Littératures.

Kristeva, J., (1969) Séméiotiké, Recherches pour une sémanalyse. Paris, Seuil, Coll. Points.

Ortel, P., (2001) La Littérature à l'ère de la photographie, Enquête sur une révolution invisible. Nîmes, Edition Jacqueline Chambon, Coll. Rayon Photo.

Riffaterre, M, (1981) «L'intertexte inconnu», in Littérature, «Intertextualités médiévales ». $\mathrm{N}^{\circ} 41, \mathrm{pp} .4-7$.

Sand, G., (1989) La Mare au diable. Paris, Presses Pocket, Lire et voir les classiques.

Scarfone, D., (1993) «Prétexte» in Trans, revue de psychanalyse, "L'empreinte, l'emprunt ». $\mathrm{N}^{\circ} 2$, pp. 9-11.

Schuerewegen, F., (1999) «Images pour mémoire», in Texte, revue de critique et de théorie littéraire. $\mathrm{N}^{\circ} 25 / 26$.

Tisseron, S., (1999) Le Mystère de la chambre claire. Photographie et inconscient, Paris, Flammarion, Coll. Champs.

\section{CORPUS}

Tlemcen ou les lieux de l'écriture, (1946) Paris, Photographies ; (1994) Revue noire.

$\underline{\text { Romans }}$

Le Sommeil d'Eve, (1989) Paris, Sindbad.

Le Désert sans détour, (1992) Paris, Sindbad.

L'Infante maure, (1994) Paris, Albin. Michel.

L'Arbre à dires, (1998) Paris, Albin Michel.

Comme un bruit d'abeilles, (2001) Paris, Albin Michel.

Simorgh, (2003) Paris, Albin Michel.

Poèmes

Ombre gardienne, (1984) Paris, Gallimard.

Le Coeur insulaire, (2000) Paris, La Différence, Coll. Clepsydre. 